\title{
NOTE
}

\section{Removal of Estrogens from Water Using Activated Carbon and Ozone}

\author{
Fumihiko Ogata, Hisato Tominaga, Hitoshi Yabutani and Naohito Kawasaki* \\ Faculty of Pharmacy, Kinki University (3-4-1 Kowakae, Higashi-Osaka, Osaka 577-8502, JAPAN)
}

\begin{abstract}
Estrogens (estron: E1; estradiol: E2; estriol: E3) are major water pollutants. For the removal of estrogens, activated carbon (AC) and ozone treatments were employed, and the chemical oxygen demand (COD) and $\mathrm{pH}$ of a single solution and multiple solution systems were investigated. The removal of estrogens increased with the amount of $\mathrm{AC}$. The percentage of removed estrogens by $\mathrm{AC}$ in the single solution system was greater than that in the ternary solution system. The estrogens were completely removed using ozone, which also reduced COD and $\mathrm{pH}$. The removal of estrogens increased the water quality and decreased the amount of organic carbon.
\end{abstract}

Key words: activated carbon, ozone treatment, estron (E1), estradiol (E2), estriol (E3)

\section{INTRODUCTION}

Estrogens are water contaminants that have been classified as endocrine disrupting compounds (EDCs). Types of EDCs include certain pesticides, plasticizers, and other industrial chemicals, as well as natural compounds such as human hormones and their breakdown products ${ }^{1)}$. Natural sources of estrone (E1) and estrogen (E2) contamination include human and mammal urine excreted in wastewater. Estrogenic activities of E1 and E2 are two or three orders of magnitude greater than those of EDCs such as nonylphenol or bisphenol $\mathrm{A}^{2)}$. Moreover, estriol(E3) is produced by the metabolism of E1. Natural estrogens are stable against decomposition by microorganisms and do not completely decompose even after several weeks. Because the release of estrogens from humans will continue to occur, removal of estrogens by treatment is important.

For the removal or degradation of estrogens, activated carbon (AC) or ozone treatment was selected. AC treatment was conducted to adsorb estrogens. This treatment, using powder or granular activated carbon, is commonly employed in sewage treatment plants ${ }^{3)}$. In contrast, ozone treatment degrades the unsaturated bonds to generate low molecular weight compounds. Moreover, ozonation and photocatalysis $\left(\mathrm{O}_{3} / \mathrm{H}_{2} \mathrm{O}_{2}\right.$ and $\left.\mathrm{O}_{3} / \mathrm{UV}\right)$ are promising techniques for removing these micropollutants ${ }^{4}$. Changes in the chemical oxygen demand (COD) with AC or ozone treatment for the removal of estrogen have been investi- gated; however, these treatments have proved ineffective.

In this study, I evaluated the effectiveness of AC and/or ozone treatment in removal of estrogens from water using a single solution and a multiple solution system. This study also investigated the amount of AC required and the changes in COD or pH in solution by ozone treatment.

\section{EXPERIMENTAL PROCEDURES}

\subsection{Materials}

The estrogens-estron(E1), estradiol(E2), and estriol (E3) - were purchased from Tokyo Chemical Industries, Co., Ltd. Quantitative measurements were conducted by high-performance liquid chromatography (HPLC: LC-10, Shimadzu) under the following conditions: column, $4.6 \mathrm{~mm}$ $\times 250$ mm Capcell Pack C18 SG120 S5 (Shiseido Co., Ltd.); mobile phase, acetonitrile:water $=55: 45$; flow rate, $1.0 \mathrm{~mL} /$ min; temperature, $40^{\circ} \mathrm{C}$; UV detection, $210 \mathrm{~nm}$.

AC with particle size between 44 and $297 \mu \mathrm{m}$ was used for chromatography. The adsorption ability of iodine, carboxyl groups, lactonic groups, and other acid groups as functional groups of $\mathrm{AC}$ were $597.71 \mathrm{mg} / \mathrm{g}, 0.25 \mathrm{mmol} / \mathrm{g}$, $0.24 \mathrm{mmol} / \mathrm{g}$, and $0.08 \mathrm{mmol} / \mathrm{g}$, respectively, and $\mathrm{pH}$ of $\mathrm{AC}$ was 4.19. Ozone was generated from oxygen gas (Iwatani GAS) using an ozone generator (Okano Works Co., Ltd.) . The generation rate of ozone (g/min), which was captured

*Correspondence to: Naohito Kawasaki, Faculty of Pharmacy, Kinki University, 3-4-1 Kowakae, Higashi-Osaka, Osaka 577-8502, JAPAN

E-mail: kawasaki@phar.kindai.ac.jp

Accepted August 8, 2011 (received for review July 26, 2011)

Journal of Oleo Science ISSN 1345-8957 print / ISSN 1347-3352 online

http://www.jstage.jst.go.jp/browse/jos/ http://mc.manusriptcentral.com/jjocs 
in $\mathrm{KI}$ solution, was determined by titration with $0.1 \mathrm{~mol} / \mathrm{L}$ $\mathrm{Na}_{2} \mathrm{~S}_{2} \mathrm{O}_{3}$.

\subsection{Removal of estrogens by $\mathrm{AC}$ and ozone treatment}

$0.5-5.0 \mathrm{mg}$ of $\mathrm{AC}$ was added to $1.5 \mathrm{~mL}$ of $20-\mathrm{mg} / \mathrm{L}$ estrogen solution. The suspensions were shaken for $1 \mathrm{~h}$ and then filtered using a $0.5-\mu \mathrm{m}$ membrane filter. The removal percentage of estrogens was calculated using the initial and equilibrium concentrations.

$200 \mathrm{~mL}$ of $100-\mathrm{mg} / \mathrm{L}$ estrogen solution was placed in an Ichinose-type gas washing bottle and aerated with ozone at a rate of $30 \mathrm{~mL} / \mathrm{min}$ for $0-90 \mathrm{~min}$. The estrogen solution was analyzed by HPLC at determined time points. In addition, COD was measured using the potassium permanganate.

\section{RESULTS AND DISCUSSION}

\subsection{Removal of estrogens by AC and ozone treatment}

The effect of dosage on the adsorption of estrogens by $\mathrm{AC}$ is shown in Fig. 1. The removal percentage of estrogens increased with the amount of AC. This result indicates that the removal percentage of estrogens depended on the amount of AC. The amount of E1 and E2 adsorbed by AC was approximately twice that of $\mathrm{E} 3$ adsorbed by $\mathrm{AC}$ in the single solution system, because the number of hydroxyl groups in E3 is larger than that in E1 or E2. The interactions between the hydroxyl groups of $\mathrm{E} 3$ and $\mathrm{AC}$ were weakly hydrophobic. In addition, the removal percentage of all three estrogens by AC in the multiple solution system was less than that in the single solution system. These results indicate that E1, E2, and E3 competed with each other for adsorption onto AC.

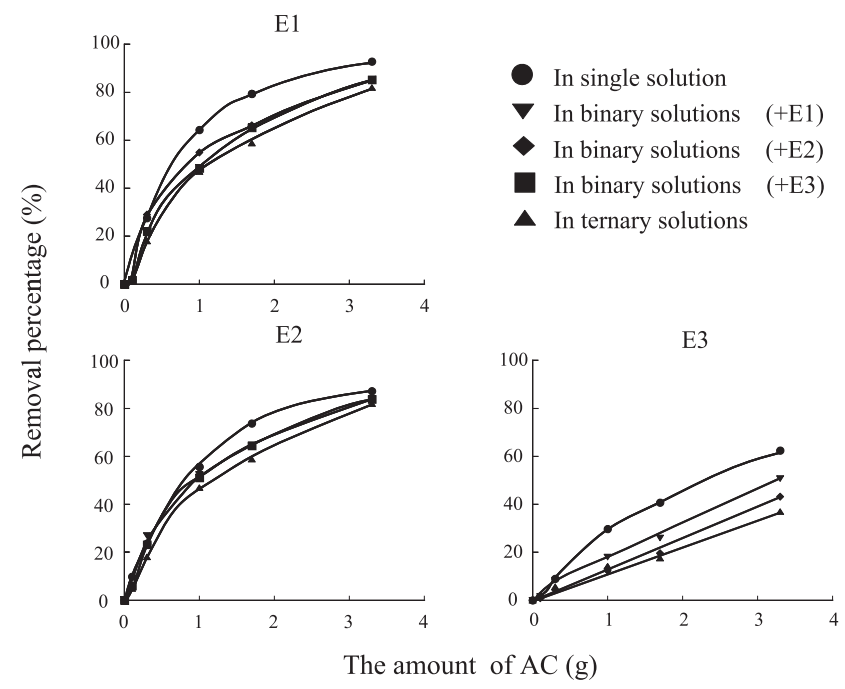

Fig. 1 Effect of dosage on adsorption of estrogens by AC.

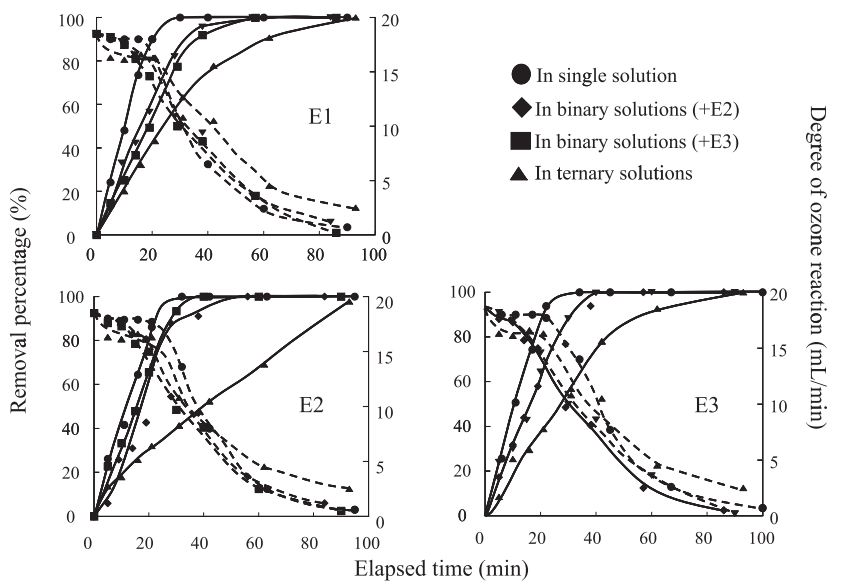

Fig. 2 Removal percentage of E1, E2, and E3 by ozone treatment. The solid line shows the removal percentage; the dotted line shows the degree of ozone reaction.

The removal percentage of estrogens by ozone treatment is shown in Fig. 2. The results indicate that complete decomposition of the estrogens occurred within $30 \mathrm{~min}$. The decomposition of estrogens by ozone treatment occurred more rapidly in the single solution system than in the multiple solutions system, which indicated competition among $\mathrm{E} 1, \mathrm{E} 2$, and $\mathrm{E} 3$. COD in the estrogen solution decreased with time during ozone treatment. Changes in the $\mathrm{pH}$ of the estrogen solution upon ozone treatment indicated that $\mathrm{pH}$ significantly decreased with time (beginning $30 \mathrm{~min}$ after initiating the ozone treatment) and remained constant thereafter. The $\mathrm{pH}$ of solutions of E1, E2, and E3 was approximately 3.5-4.0 for the single and multiple solution systems.

\section{CONCLUSION}

The removal percentage of estrogens by AC depended on the amount of AC. In a single solution system, the removal percentage of $\mathrm{E} 3$ by $\mathrm{AC}$ was less than that of $\mathrm{E} 1$ or E2. The removal of estrogens by AC in the single solution system was greater than that in a ternary solution system. Estrogens were completely degraded by ozone treatment. The removal of E3 was faster than that of E1 or E2. COD decreased with ozone treatment. The difference in the hydroxyl group structures of E1, E2, and E3 affected the removal of estrogens by $\mathrm{AC}$ or degradation by ozone treatment.

\section{ACKNOWLEDGEMENTS}

This work was financially supported by "Antiaging Center Project" for Private Universities from the Ministry 
of Education, Culture, Sports, Science and Technology, 2008-2012.

\section{References}

1) Irmak, S.; Erbatur, O.; Akgerman, A. Degradation of $17 \beta$-estradiol and bisphenol A in aqueous medium by using ozone and ozone/UV techniques. J. Hazard. Mat. B. 126, 54-62 (2005).

2) Routledge, E. J.; Sumpter, J. P. Estrogenic activity of surfactants and some of their degradation products assessed using a recombinant yeast screen. Environ. Toxicol. Chem. 15, 241-248(1996).

3) Rowsell, V. F.; Gheng, Pang, D. S.; Tsafou, F.; Voulvoulis, N. Removal of steroid estrogens grom wastewater using granular activated carbon: Comparison between virgin and reactivated carbon. Water Environ. Res. 81, 394-400 (2009).

4) Bila, D.; Montalvao, A. F.; Azevedo, D. O.; Dezotti, M. Estrogenic activity removal of $17 \beta$-estradiol by ozonation and identification of by-products. Chemosphere 69, 736-746 (2007). 